\title{
Fluorescence and Diffuse Reflectance Spectroscopy for early cancer detection using a new strategy towards the development of a miniaturized system
}

\author{
D. S. Ferreira, P. J. G. Coutinho, E. M. S. Castanheira, J. H. Correia, and G. Minas
}

\begin{abstract}
This paper describes the design of a miniature, costeffective spectroscopy system for assessing tissue biochemical and morphological information using a few wavelengths. This instrument will integrate thin-film optical filters and silicon photodiodes, avoiding the use of a spectrograph and optical fibers. The components in the set-up design are described. The feasibility of using only 16 wavelengths to accurately extract tissue properties is confirmed on physical tissue models. Also, the suitable spectral performance of several optical filters for the selection of these wavelengths is demonstrated. The small size of this device will enable implementation in an endoscopic capsule.
\end{abstract}

\section{INTRODUCTION}

$\mathrm{C}$ ANCERS of epithelial origin usually progress through increasing grades of dysplasia. Early detection of dysplasia is essential for managing cancer, since the chances of successful treatment increase significantly when the disease is diagnosed at an early, non-invasive stage. However, dysplastic lesions are not always endoscopically visible, thus requiring several random, unnecessary tissue biopsies to be taken. Optical methods may overcome some limitations of current screening methods [1-3]. Diffuse reflectance spectroscopy (DRS) and intrinsic fluorescence spectroscopy (IFS) have shown great ability for the detection of dysplasia, by exhibiting different spectral features that can be correlated with normal and diseased tissue. These modalities provide quantitative information about biochemical and structural tissue attributes (tissue parameters), from which objective diagnostic algorithms are developed [3].

Diffuse reflectance spectra from tissues are used to extract information about hemoglobin concentration and saturation, light scattering parameters, and other tissue characteristics, using a well-developed model based on the diffusion approximation of light propagation in tissue. This method is known as DRS and provides information about the morphology and biochemistry of bulk tissue [4]. Intrinsic fluorescence is the fluorescence unaffected by tissue

D. S. Ferreira, J. H. Correia and G. Minas are with the Industrial Electronics Department, University of Minho, Campus de Azurém, Guimarães, 4800-058, Portugal. (corresponding author: debora@ mit.edu)

P. J. G. Coutinho and E. M. S. Castanheira are with the Centre of Physics (CFUM), University of Minho, Campus de Gualtar, Braga, 4710057, Portugal. scattering and absorption, and is obtained using the diffusely reflected light to remove spectral distortions. The relative contributions of endogenous tissue fluorophores (e.g., NADH and collagen) can be extracted from the intrinsic fluorescence, being the method known as IFS. The concentrations of these fluorophores are dependent on the tissue disease state. Frequently, biochemical changes precede morphological changes within the tissue, which opens the possibility for a very early detection of dysplasia [5].

Clinical instruments designed to perform DRS and IFS usually employ optical fibers for light delivery and collection. This can be a drawback since regular optical fibers only collect a small portion of the reemitted signal, thus requiring high quantum efficiency detectors, such as charge coupled device cameras (CCD's). Also, they integrate costly and sophisticated illumination equipment (xenon arc lamps, UV lasers). For this reason, the development of a simpler, miniature, cost-effective spectroscopy system without the need for optical fibers, spectrograph or CCD cameras could potential increase the collection efficiency and improve throughput. A few groups have attempted to develop instruments with some of these features using LEDs for illumination and photodiodes for detection [6-8].

As a long-term goal we propose the development of a miniaturized spectroscopy system to be integrated on an endoscopic capsule for the detection of dysplasia in the gastrointestinal tract. This system uses thin-film optical filters and low-cost silicon photodiodes for the selection and detection of several light wavelengths significant for diagnosis. Ultraviolet and white-light LEDs are used as the illumination sources for fluorescence and diffuse reflectance measurements, respectively.

This report describes the simple design of the instrument. As a first step towards the final goal, wavelength reduction simulations are performed to evidence the feasibility of replacing the spectrograph by several thin-film optical filters, and the use of just a few wavelengths to accurately quantify tissue parameters. Physical tissue models with well defined optical properties were used for this feasibility study. The design and performance of thin-film optical filters is illustrated. 


\section{INSTRUMENTATION}

A miniaturized spectroscopy system will be designed to be integrated in an endoscopic capsule. The instrument is based on white-light and UV LEDs, and optical microsensors (thin-film optical filters together with low-cost silicon photodiodes). The firsts will be used as the illumination sources for diffuse reflectance and fluorescence measurements, and the second to select and detect specific light wavelengths. The use of these components obviates the need for costly, bulky and sophisticated illumination and detection equipment (high-power xenon arc lamp, UV laser, CCD camera, spectrograph), and optical fibers for light delivery and collection.

This microsystem will add essential diagnostic functions to the current endoscopic capsules, taking advantage of the commercial available capsule platform (such as the battery, wireless data transmission, antenna, etc.). Figure 1 depicts the several components of the miniaturized spectroscopy system.

\section{A. Wavelength Reduction Simulations on Physical Tissue Models}

The replacement of the spectrograph by a series of optical filters was investigated in the 350 to $750 \mathrm{~nm}$ spectral range. The capability of a spectroscopy system to extract tissue information with accuracy using just a few wavelengths was evaluated with a series of experiments on physical tissue models ("phantoms") with known scattering and absorption parameters. The phantoms consist of mixtures of intralipid and hemoglobin (Sigma Aldrich Co.) at various concentrations, and water. The intralipid and the hemoglobin are used for scattering and absorbing, respectively.

Two groups of phantoms were measured by diffuse reflectance spectroscopy: the first with different concentrations of absorber and a fixed intralipid concentration; the second with a variable scatterer concentration and a fixed hemoglobin amount. A solid sample of $\mathrm{BaSO}_{4}$ was used as a reflectance standard. The UV-3101PC spectrophotometer, from Shimadzu, was used to measure the diffuse reflectance spectra.

DRS is used for the extraction of tissue quantitative information. By fitting the reflectance spectrum to the model described by Zonios et al. [4], four tissue optical parameters are extracted: $A$, the reduced scattering coefficient at the reference wavelength; $B$, related to the average scatter size; $c H b$, the concentration of hemoglobin; and $\alpha$, the oxygen saturation of hemoglobin.

Several combinations of 16 wavelengths were simulated. The combination that provided the best results, in terms of tissue parameters extraction, comprises the following discrete data points: $350,370,380,400,420,450,480,510$, $540,560,580,600,620,650,700$, and $750 \mathrm{~nm}$. These 16 wavelengths will be selectively detected using one stack of $\mathrm{TiO}_{2}$ and $\mathrm{SiO}_{2}$ thin-films, placed on top of the photodiode, as will be detailed on the next subsection. The remaining spectral values will be obtained by interpolation within the range of the discrete data set.

\section{B. Thin-film Optical Filters}

A thin-film optical filter array (composed by 16 filters) will be deposited by ion beam deposition on top of photodiodes. The filtering system, based on Fabry-Perot thin-film optical resonators, should be designed to yield a narrow pass-band around the selected wavelengths. With this optical filter array only a white light and a UV source for illumination will be needed, thus avoiding a full spectrograph.

The filters consist of two flat parallel mirrors composed of a stack of $\mathrm{TiO}_{2}$ and $\mathrm{SiO}_{2}$ thin-films, with a $\mathrm{SiO}_{2}$ resonance cavity in the middle. $\mathrm{TiO}_{2}$ and $\mathrm{SiO}_{2}$ have been selected because of the IC compatibility and the well characterization of their deposition process. In addition, the refractive index of $\mathrm{SiO}_{2}$ is almost wavelength independent for the spectral band between $350 \mathrm{~nm}$ and $750 \mathrm{~nm}$.

\section{RESULTS}

\section{A. Tissue phantoms study}

In order to experimental determine the accuracy of a spectroscopy system to extract tissue properties using only 16 wavelengths, several DRS measurements were performed on tissue phantoms, in a range of absorbing and scattering concentrations. The collected spectra were normalized by the reflectance spectra measured from the standard, to correct for the wavelength dependent response of the system.

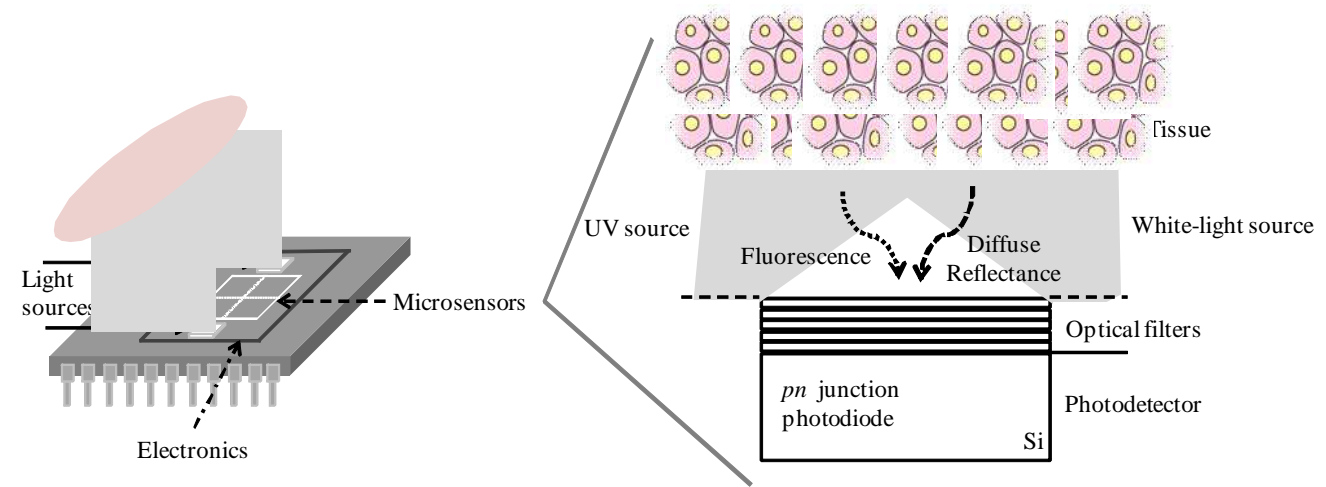

Fig. 1. Miniaturized spectroscopy system with LEDs as light sources, optical filters and photodetector for wavelength selection and detection, respectively (not scaled). 
Figure 2 shows the diffuse reflectance spectra taken from the same position on different phantoms using the full wavelength range, and using only 16 selected wavelengths. These 16 intensities are the signal that would be read by the photodiode array. DRS is then used to extract the tissue optical parameters by fitting each measured reflectance spectrum (solid lines) to the model described by Zonios et al. (dashed lines).

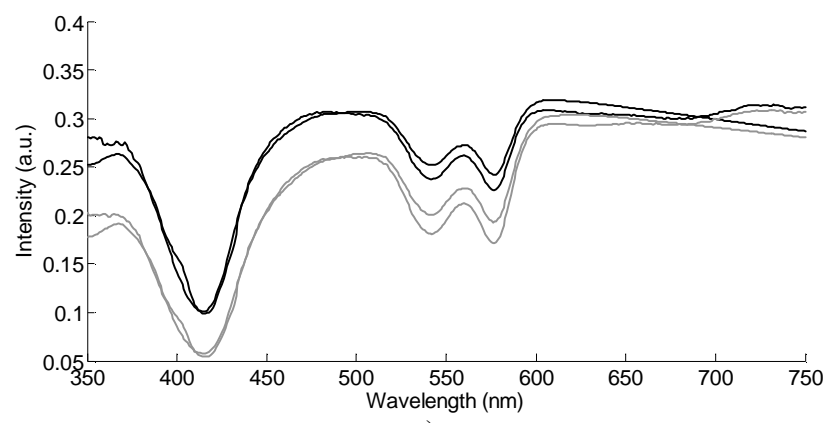

a)

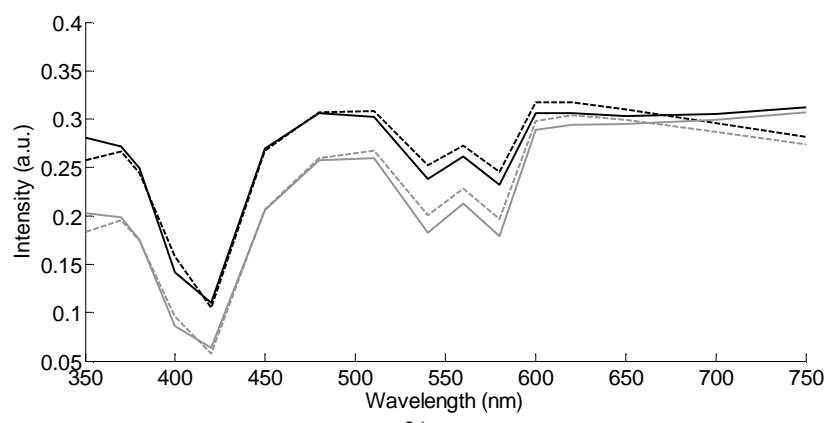

b)

Fig. 2. Reflectance spectra measured from different tissue phantoms (phantom 1: black line; phantom 2: grey line): (a) using the full wavelength range; (b) and only 16 wavelengths. The best fit spectra according to the model of Zonios et al. are also plotted (dashed lines).

Table 1 presents the extracted values for parameters $A, B$, $\alpha$, and $c H b$, from different phantoms. As expected, the value of $A$ increases with intralipid concentration. The value of $\mathrm{cHb}$ is very similar to the expected value with less than $10 \%$ difference. The use of the full wavelength range to measure diffuse reflectance or the use of only 16 wavelengths seems to provide very comparable results in terms of optical properties in tissue physical models. These results point out that it is not necessary to use the full $350-750 \mathrm{~nm}$ spectrum to extract optical properties with sufficient accuracy.

The phantoms presented in this study are simplified compared to the composition of human tissue. However, hemoglobin, which is a very important parameter to differentiate normal from malignant tissues, is considered the most important absorber in the visible region of the spectra for human tissue, and its concentration can be extracted with good accuracy using just a few wavelengths. In the same way, other physiological parameters, such as $\mathrm{NADH}$ and collagen, may also be quantified using just a few wavelengths.

\section{B. Optical Filtering System}

The results of the previous section have shown the possibility of using only 16 wavelengths to extract tissue optical properties. The selection of these wavelengths can be achieved using an optical filter array. This array is composed by four groups of four optical filters to cover different spectral ranges: $350-400 \mathrm{~nm} ; 420-510 \mathrm{~nm} ; 540-600 \mathrm{~nm}$; and 620-750 nm.

Thin-film optics software TFCalc ${ }^{\mathrm{TM}} 3.5$ was used for the structural optimization of the optical filters. Simulation results show that a multilayer stack of $\mathrm{TiO}_{2}$ and $\mathrm{SiO}_{2}$ thinfilms for the dielectric mirrors with a $\mathrm{SiO}_{2}$ resonance cavity is the best option in terms of optical characteristics and fabrication process. For each of the mentioned groups, the optical filters are composed by 11 layers of $\mathrm{TiO}_{2}$ and $\mathrm{SiO}_{2}$, and can be easily tuned to a different wavelength by adjusting only the thickness of the 6th layer (the $\mathrm{SiO}_{2}$ resonance cavity). This $\mathrm{SiO}_{2}$ thickness, in each group, increases $9 \mathrm{~nm}$ to move the wavelength peak $10 \mathrm{~nm}$.

In Figure 3 the simulated transmittances of 8 Fabry-Perot optical filters are presented, with the layer stacks described in Table 2.

TABLE 1 REFLECTANCE PARAMETERS MEASURED FROM TISSUE PHANTOMS WITH DIFFERENT HEMOGLOBIN (HB) AND INTRALIPID CONCENTRATIONS

\begin{tabular}{|c|c|c|c|c|c|c|c|c|c|c|}
\hline \multirow[b]{2}{*}{ Phantoms } & \multirow[b]{2}{*}{$\begin{array}{c}\text { Intralipid } \\
\text { mass } \\
\text { concentration }\end{array}$} & \multirow[b]{2}{*}{$\begin{array}{c}\mathrm{Hb} \\
\text { concentration }\end{array}$} & \multicolumn{4}{|c|}{ Full Spectrum } & \multicolumn{4}{|c|}{16 Wavelengths } \\
\hline & & & $\begin{array}{c}c H b \\
(\mathrm{mg} / \mathrm{mL})\end{array}$ & $A$ & $B$ & $\alpha$ & $\begin{array}{c}c H b \\
(\mathrm{mg} / \mathrm{mL})\end{array}$ & $A$ & $B$ & $\alpha$ \\
\hline 1 & $0.5 \%$ & 0.5 & 0.498 & 0.914 & 0.673 & 1.0 & 0.519 & 0.903 & 0.771 & 1.0 \\
\hline 2 & $0.5 \%$ & 1.0 & 0.944 & 0.887 & 0.538 & 1.0 & 1.013 & 0.873 & 0.704 & 1.0 \\
\hline 3 & $1 \%$ & 0.5 & 0.480 & 1.787 & 0.546 & 1.0 & 0.499 & 1.759 & 0.648 & 1.0 \\
\hline 4 & $1 \%$ & 1.0 & 0.988 & 1.782 & 0.559 & 1.0 & 1.075 & 1.752 & 0.742 & 1.0 \\
\hline
\end{tabular}


TABLE 2

MAXIMUM TRANSMISSION PEAK AND LAYER THICKNESS OF 8 FABRY-PEROT OPTICAL FILTERS.

\begin{tabular}{|c|c|c|c|c|c|c|c|c|c|}
\hline & & \multicolumn{8}{|c|}{ Wavelength transmission peak (nm) } \\
\hline & & 420 & 4450 & 480 & 2510 & 2540 & 2560 & 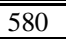 & 600 \\
\hline & & \multicolumn{8}{|c|}{ Layer thickness (nm) } \\
\hline \multirow{5}{*}{ Mirror } & $\overline{\mathrm{TiO}_{2}}$ & 42 & 42 & 42 & 42 & $\overline{58}$ & $\overline{258}$ & $\overline{58}$ & 58 \\
\hline & $\mathrm{SiO}_{2}$ & 74 & 74 & 74 & 74 & 92 & 92 & 92 & 92 \\
\hline & $\mathrm{TiO}_{2}$ & 42 & 42 & 42 & 42 & 58 & 58 & 58 & 58 \\
\hline & $\mathrm{SiO}_{2}$ & 74 & 74 & 74 & 74 & 92 & 92 & 92 & 92 \\
\hline & $\mathrm{TiO}_{2}$ & 42 & 42 & 42 & 42 & 58 & 58 & 58 & 58 \\
\hline \multirow[t]{3}{*}{ Cavity } & $\mathrm{SiO}_{2}$ & 127 & 154 & 181 & 199 & 166 & 184 & 202 & 220 \\
\hline & $\mathrm{TiO}_{2}$ & 42 & 42 & 42 & 42 & 58 & 58 & 58 & 58 \\
\hline & $\mathrm{SiO}_{2}$ & 74 & 74 & 74 & 74 & 92 & 92 & 92 & 92 \\
\hline \multirow[t]{3}{*}{ Mirror } & $\mathrm{TiO}_{2}$ & 42 & 42 & 42 & 42 & 58 & 58 & 58 & 58 \\
\hline & $\mathrm{SiO}_{2}$ & 74 & 74 & 74 & 74 & 92 & 92 & 92 & 92 \\
\hline & $\mathrm{TiO}_{2}$ & 42 & 42 & 42 & 42 & 58 & 58 & 58 & 58 \\
\hline
\end{tabular}

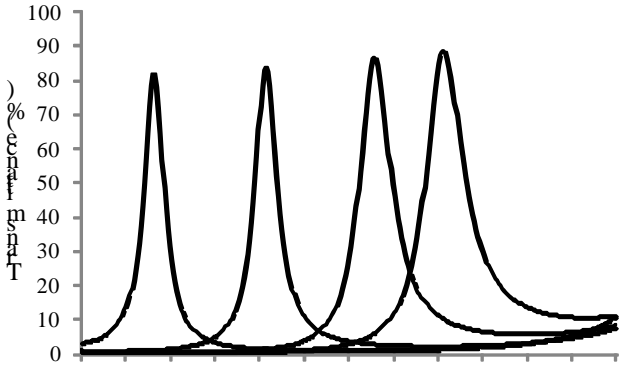

400413425438450463475488500513525538550

Wavelength $(\mathrm{nm})$

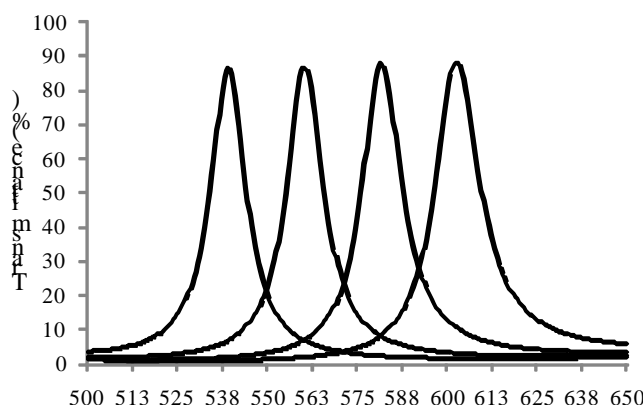

Wavelength $(\mathrm{nm})$

Fig. 3. Simulated transmittance spectra for 8 Fabry-Perot optical filters.

\section{CONCLUSION}

This study demonstrated that it is possible to extract tissue information accurately using a small number of wavelengths. These results are a very important step towards the development of a much smaller, high throughput system, compared with the conventional clinical spectroscopy instruments.

Overall, the results showed the feasibility of replacing the spectrograph by a series of thin-film optical filters. Also, with UV and white-light LEDs as illumination sources and photodiodes as the detector, the device would then be truly miniaturized, while still achieving comparable performance in the extraction of tissue optical properties. The fabrication of the entire system and its preliminary results will be discussed elsewhere.

\section{ACKNOWLEDGMENT}

This research was supported by the Portuguese Foundation of Science and Technology and the MIT|Portugal Program (SFRH / BD / 38978 / 2007).

\section{REFERENCES}

[1] D. Antonioli, "Esophagus", in Pathology of Incipient Neoplasia, 3rd ed., D. Henson and J. Albores-Saavedra, Ed. W.B. Saunders Company, 1993, pp. 64-83.
[2] J. W. Tunnell, A. E. Desjardins, L. Galindo, I. Georgakoudi, S. A. McGee, J. Mirkovic, M. G. Mueller, J. Nazemi, F. T. Nguyen, A. Wax, Q. G. Zhang, R. R. Dasari, and M. S. Feld, "Instrumentation for multimodal spectroscopic diagnosis of epithelial dysplasia," Technol. Cancer Res. Treat., vol. 2, 2003, pp. 505-514

[3] C. Yu, C. Lau, G. O’Donoghue, J. Mirkovic, S. McGee, L. Galindo, A. Elackattu, E. Stier, G. Grillone, K. Badizadegan, R. R. Dasari, and M. S. Feld, "Quantitative spectroscopic imaging for noninvasive early cancer detection”, Optics Express, vol. 16, 2008, pp. 16227-16239.

[4] G. Zonios, L. T. Perelman, V. M. Backman, R. Manoharan, M. Fitzmaurice, J. Van Dam, and M. S. Feld, "Diffuse reflectance spectroscopy of human adenomatous colon polyps in vivo," Appl. Opt., vol. 38, 1999, pp. 6628-6637.

[5] M. G. Muller, I. Georgakoudi, Q. G. Zhang, J. Wu, and M. S. Feld, "Intrinsic fluorescence spectroscopy in turbid media: disentangling effects of scattering and absorption," Appl. Opt., vol. 40, 2001, pp. 4633-4646.

[6] J. W. Feather, D. J. Ellis, G. Leslie, "A portable reflectometer for the rapid quantification of cutaneous haemoglobin and melanin", Phys. Med. Biol., vol. 33, 1988, pp. 711-722.

[7] B. Yu, J. Y. Lo, T. F. Kuech, G. M. Palmer, J. E. Bender, N. Ramanujam, "Cost-effective diffuse reflectance spectroscopy device for quantifying tissue absorption and scattering in vivo", Journal of Biomedical Optics, vol. 13, 2008.

[8] J. Y. Lo, B. Yu, H. L. Fu, J. E. Bender, G. M. Palmer, T. F. Kuech, N. Ramanujam, "A strategy for quantitative spectral imaging of tissue absorption and scattering using light emitting diodes and photodiodes", Optics Express, vol. 17, 2009, pp. 1372-1384. 